\title{
Impact of ABO Blood Types on Survival after Pancreatectomy for Pancreatic Cancer ${ }^{*}$
}

\author{
Tadashi Kayashima $^{1}$, Masafumi Nakamura ${ }^{1}$, Kenoki Ohuchida ${ }^{1,2}$, Syunichi Takahata ${ }^{1}$, Kohei Nakata $^{1}$, \\ Naoki Ikenaga ${ }^{1}$, Lin Cui ${ }^{1}$, Shingo Kozono ${ }^{1}$, Kazuhiro Mizumoto ${ }^{1,3}$, Masao Tanaka ${ }^{1 \#}$ \\ ${ }^{1}$ Department of Surgery and Oncology, Graduate School of Medical Sciences, Kyushu University, Fukuoka, Japan; ${ }^{2}$ Department of \\ Advanced Medical Initiatives, Graduate School of Medical Sciences, Kyushu University, Fukuoka, Japan; ${ }^{3}$ Kyushu University Hos- \\ pital Cancer Center, Fukuoka, Japan. \\ Email: \#masaotan@med.kyushu-u.ac.jp
}

Received July $18^{\text {th }}, 2013$; revised August $17^{\text {th }}, 2013$; accepted August 25 ${ }^{\text {th }}, 2013$

Copyright (c) 2013 Tadashi Kayashima et al. This is an open access article distributed under the Creative Commons Attribution License, which permits unrestricted use, distribution, and reproduction in any medium, provided the original work is properly cited.

\begin{abstract}
Background/Purpose: A recent genome-wide association study (PanScan) identified significant association between the ABO gene locus and the risk of pancreatic cancer. The aim of this study was to analyze survival of pancreatic cancer patients who underwent surgical resection based on serotype-defined ABO blood types. Methods: In terms of ABO blood types and gemcitabine-based adjuvant therapy, we investigated the survival of 153 patients who underwent pancreatectomy for pancreatic cancer at the Department of Surgery and Oncology, Kyushu University (Fukuoka, Japan). Results: Among the four blood type groups, the O serotype group (median survival time (MST): 47.9 months) showed the best prognostic outcome. The A serotype group (MST: 22.5 months) showed the second best prognostic outcome, followed by the AB serotype group (MST: 20.4 months). The B serotype group (MST: 15 months) showed the worst prognostic outcome when considering the MST after the surgical resection. Next, we examined the effect of adjuvant chemotherapy with gemcitabine based on the ABO blood types. The A serotype group showed the greatest improvement in prognosis by adjuvant therapy with gemcitabine after the surgical resection. The other three serotype groups showed no significant differences in the prognostic outcomes between subgroups with and without gemcitabine therapy. Conclusions: The present data suggest the possibility that ABO blood types are prognostic factors for pancreatic cancer patients after surgical treatment and are also predictive factors for the chemosensitivity to gemcitabine of pancreatic cancer patients after pancreatectomy.
\end{abstract}

Keywords: Pancreatic Cancer; ABO Blood Type; Survival; Gemcitabine; Adjuvant Chemotherapy

\section{Introduction}

Pancreatic cancer is the fourth leading cause of cancerrelated death in the United States, and has the lowest survival rate for any solid tumor [1,2]. Owing to a lack of early detection methods and an absence of effective biomarkers, patients are usually diagnosed at late stages with a 5-year survival rate of $<5 \%$ [1]. The first-line agent gemcitabine produces some clinical benefit in the advanced setting, but yields limited disease control, with $<15 \%$ of patients remaining progression-free at 6 months

\footnotetext{
*Supported in part by a Grant-in-Aid from the Ministry of Education, Culture, Sports, Science and Technology of Japan. The current address of Masafumi Nakamura is Department of Digestive Surgery, Kawasaki Medical School, Kurashiki, Japan.

${ }^{\#}$ Corresponding author.
}

after diagnosis [3,4].

For several decades, roles of the ABO blood group antigens have been suspected in the development of certain malignancies. A recent genome-wide association study (PanScan) identified significant association of the ABO gene locus with the risk of pancreatic cancer, but the influence of the specific ABO serotypes remains unknown [5].

To date, there are some studies that have investigated the correlations between certain gene expression levels and chemosensitivity to gemcitabine in pancreatic cancer patients [6-13]. However, to our knowledge, there are no reports regarding the relationships between chemosensitivity and $\mathrm{ABO}$ blood types in pancreatic cancer after surgical resection. 
In the present study, we analyzed the survival and effects of gemcitabine based on serotype-defined ABO blood types in 153 patients who underwent surgical resection at the Department of Surgery and Oncology, Kyushu University (Fukuoka, Japan). Our data suggest that the $\mathrm{O}$ serotype is associated with significantly longer survival of the patients than other ABO blood groups and that the A serotype is correlated with better effects of gemcitabine on survival of the patients after surgical resection.

\section{Methods}

\subsection{Patients}

The medical records of 153 patients who underwent surgical resection for pancreatic cancer at the Department of Surgery and Oncology, Kyushu University (Fukuoka, Japan), between 27 January 1992 and 19 March 2010 were retrospectively reviewed. The $\mathrm{ABO}$ blood types were as follows: A serotype, 75 patients; O serotype, 33 patients; B serotype, 26 patients; AB serotype, 19 patients. The characteristics of the patients are shown in Table 1.

\subsection{Statistical Analysis}

All statistical analyses were carried out using JMP 8.0.1 software (SAS Institute, Cary, NC). Survival curves were calculated by the Kaplan-Meier method, and differences between the curves were analyzed by the log-rank test. All differences were considered to be statistically significant for values of $P<0.05$.

\section{Results}

\subsection{Survival Curves Based on the ABO Blood types}

The survival curves based on the serotype-defined ABO blood types are shown in Figure 1(a). The respective 5-year survival rate and median survival time (MST) for each of the four serotype groups were as follows: O serotype group, $47.1 \%$ and 47.9 months; A serotype group, $22.6 \%$ and 22.5 months; $\mathrm{AB}$ serotype group, $0 \%$ and 20.4 months; and B serotype group, $25.2 \%$ and 15 months.

In a comparison of the prognoses in the $\mathrm{O}$ serotype group and a combined non-O serotype group, the 5-year survival rate and MST in the O serotype group (47.1\% and 47.9 months, respectively) were significantly higher and longer, respectively, than those in the non-O serotype group (20.5\% and 22.5 months, respectively; $P=$ 0.0276, log-rank test) (Figure 1(b)).

There was no distance by R0/R1, 2 ( $P=0.790$ Chisquare test), and with radiotherapy (post or preoperative) or without radiotherapy ( $P=0.152$ Chi-square test), operative method (DP/PD and PpPD/TP, $P=0.930$ Chisquare test), staging according UICC TNM $(0 \sim$ II/III IV, $P=0.105$ Chi-square test), but there was significant difference by lymph nodes negative or positive $(P<0.01$ Chi-square test).

In addition, the median disease-free survival time and 5 -year disease-free survival rate in the serotype $\mathrm{O}$ group tended to be longer and higher, respectively, than those in the non-O serotype group (Figure 1(c); mean diseasefree survival time: 42 months vs. 14.5 months; 5-year disease-free survival rate: $10.6 \%$ vs. $7.3 \%$; $P=0.066$, log-rank test), but there was no significant difference.

\subsection{Effects of Gemcitabine Adjuvant Chemotherapy Based on ABO Blood Types}

Next, we compared the effect of chemotherapy with gemcitabine on survival of the pancreatic cancer patients among the ABO blood type groups. Two patients in the A serotype group were excluded from the analysis because their treatments after the surgical resection were unknown. Consequently, 151 patients were analyzed for their survival (Table 1). The A serotype group showed

Table 1. Characteristics of the patients based on the serotype-defined ABO blood types.

\begin{tabular}{|c|c|c|c|c|}
\hline \multirow[t]{2}{*}{ Characteristic } & \multicolumn{4}{|c|}{ Serologic-derived ABO blood type } \\
\hline & $\mathbf{O}$ & A & AB & B \\
\hline No. of patients & 33 & 75 & 19 & 26 \\
\hline Mean age & $63.5 \pm 10.3$ & $64.2 \pm 10.5$ & $64.1 \pm 7.6$ & $69.9 \pm 7.4$ \\
\hline Female gender $(\%)$ & $36.4 \%$ & $38.2 \%$ & $21.1 \%$ & $30.8 \%$ \\
\hline Staging according to UICC TNM $(0 \sim$ II/III IV $)$ & $32 / 1$ & $71 / 5$ & $18 / 1$ & $23 / 3$ \\
\hline With Gemcitabine treatment after the surgical resection & 21 & 37 & 10 & 15 \\
\hline Mean age & $65 \pm 9.3$ & $61.8 \pm 10.9$ & $62.1 \pm 8.3$ & $70.7 \pm 7.3$ \\
\hline Female gender (\%) & $33.3 \%$ & $37.8 \%$ & $30 \%$ & $26.7 \%$ \\
\hline Staging according to UICC TNM $(0 \sim$ II/III $\sim$ IV $)$ & $21 / 0$ & $34 / 3$ & $9 / 1$ & $13 / 2$ \\
\hline
\end{tabular}




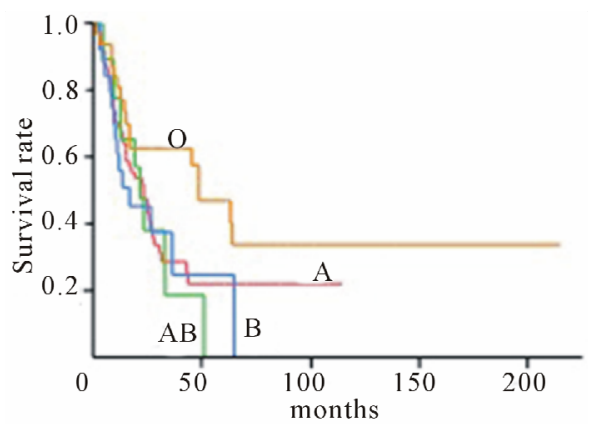

(a)

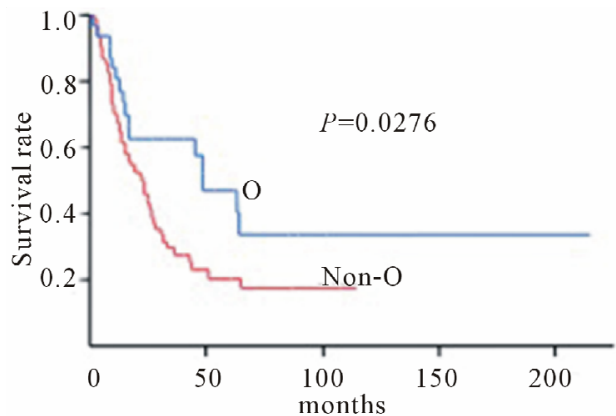

(b)

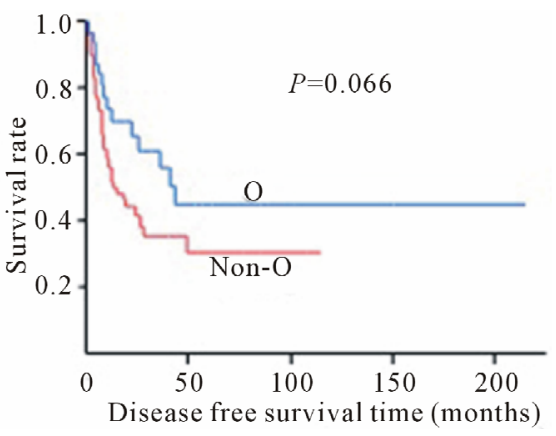

(c)

Figure 1. Survival curves based on the ABO blood serotypes of pancreatic cancer patients after surgical resection. (a) Survival curves based on the ABO blood serotypes. Among the four blood serotypes, the $O$ serotype group showed the best prognostic outcome after surgical resection of pancreatic cancer $(n=33$; 5-year survival rate: 47.1\%; MST: 47.9 months). The A serotype group showed the second best prognostic outcome (n = 75; 5-year survival rate: $22.6 \%$; MST: 22.5 months), followed by the AB serotype group ( $n=19$; 5-year survival rate: 0\%; MST: 20.4 months). The B serotype group showed the worst prognostic outcome ( $=26$; 5-year survival rate: $25.2 \%$; MST: 15 months) when considering the MST after the surgical resection. (b) Survival curves based on the $O$ serotype group and a combined non-O serotype group. The MST and 5-year survival rate were significantly longer and higher, respectively, in the $O$ serotype group $(n=33)$ than in the non-O serotype group $(n=120)$ (MST: 47.9 months vs. 22.5 months; 5-year survival rate: $47.1 \%$ vs. $20.5 \%$; $P=0.0276$, log-rank test). (c) Disease-free survival curves based on the $O$ serotype group and the non- $O$ serotype group. The median disease-free survival time and 5 -year disease-free survival rate also tended to be longer and higher, respectively, in the $O$ serotype group than in the non-O serotype group (mean disease-free survival time: 42 months vs. 14.5 months; 5-year disease-free survival rate: $10.6 \%$ vs. $7.3 \% ; P=0.066$, log-rank test), but the difference was not significant.

the greatest effect of gemcitabine treatment (Figure 2(a); gemcitabine treatment group $(n=37)$ vs. no gemcitabine treatment group $(\mathrm{n}=36)$, 5-year survival rate: $39.7 \%$ vs. 9.6\%; MST: 30.5 months vs. 10.4 months; $P=0.002$ ). The other three serotype groups showed no significant differences in the prognosis between subgroups with and without gemcitabine treatment (Figure 2(b)-(d)).

\section{Discussion}

In the present study, we found that the MST and 5-year survival rate of the $\mathrm{O}$ serotype group of patients after pancreatic cancer resection were significantly longer and higher, respectively, than those in the other serotype groups, and that adjuvant gemcitabine therapy for pancreatic cancer was more effective for the A serotype group of the patients compared with the other serotype groups.

Consistent with our findings, PanScan, a recent genome-wide association study, identified significant asso- ciations between the $\mathrm{ABO}$ gene locus and the risk of pancreatic cancer [5]. In addition, another recent study of patients who underwent a potentially curative operation in China showed that the mean survival time of patients with blood group $\mathrm{O}$ was significantly longer than that of patients with non-O blood groups (16.0 months vs. 11.0 months, respectively, $P=0.001$, log-rank test) [14]. Their study showed an evidence of an association between blood groups and the risk of the development and progression of pancreatic cancer. However, the MSTs of their patients with $\mathrm{O}$ and non-O blood groups were much shorter than those of our patients. The exact reason for these differences remains unknown.

The ABO human blood group antigens are glycoproteins expressed on the surface of red blood cells and the cells in several other tissue types. For example, the ABO antigens are highly expressed on the surface of epithelial cells in the gastrointestinal, bronchopulmonary, and urogenital tracts $[15,16]$. Taking our findings and the previ- 


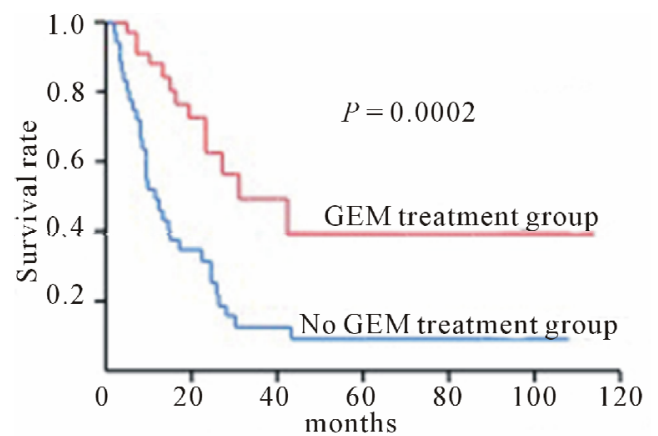

(a)

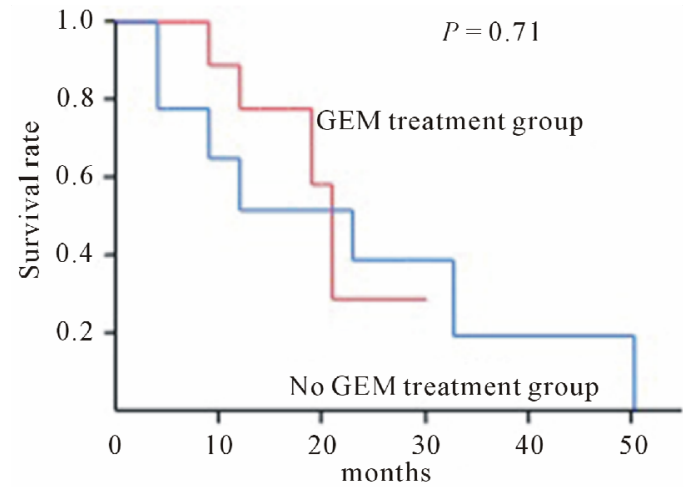

(c)

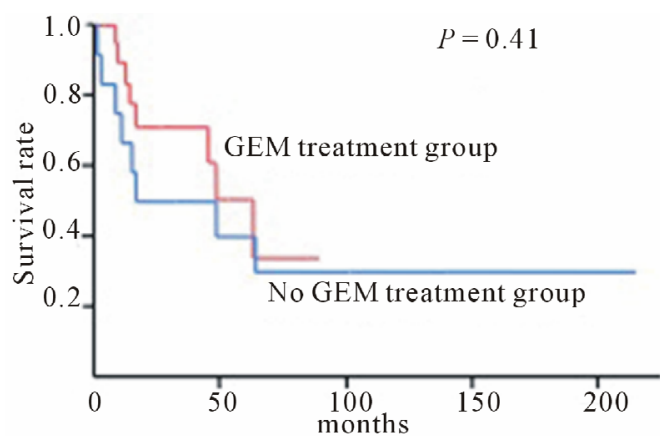

(b)

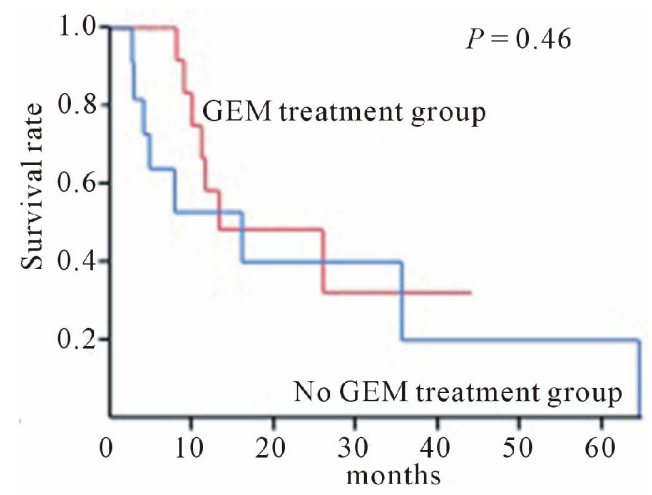

(d)

Figure 2. Survival of pancreatic cancer patients after surgical resection with and without adjuvant gemcitabine chemotherapy based on the ABO blood serotypes. (a) Survival curves based on subgroups with and without gemcitabine treatment after surgical resection in the A serotype group (gemcitabine treatment group: $\mathbf{n}=37,5$-year survival rate: $39.7 \%$, MST: 30.5 months; no gemcitabine treatment group: $n=36$, 5-year survival rate: $9.6 \%$, MST: 10.4 months; $P=0.0002$, log-rank test). (b) Survival curves based on subgroups with and without gemcitabine treatment after surgical resection in the $O$ serotype group (gemcitabine treatment group: $n=21$, 5-year survival rate: 50.8\%, MST: 62.1 months; no gemcitabine treatment group: $n=$ 12, 5-year survival rate: $\mathbf{4 0 \%}$, MST: 39.8 months; $P=\mathbf{0 . 4 1}$, log-rank test). (c) Survival curves based on subgroups with and without gemcitabine treatment after surgical resection in the AB serotype group (gemcitabine treatment group: $\mathbf{n}=10$, 5-year survival rate: $0 \%$, MST: 20.4 months; no gemcitabine treatment group: $\mathbf{n}=\mathbf{9}$, 5-year survival rate: $0 \%$, MST: 17.5 months; $P=0.71$, log-rank test). (d) Survival curves based on subgroups with and without gemcitabine treatment after surgical resection in the B serotype group (gemcitabine treatment group: $n=15$, 5-year survival rate: $0 \%$, MST: 13.1 months; no gemcitabine treatment group: $n=11,5$-year survival rate: $19.9 \%$, MST: 15 months; $P=0.46$, log-rank test).

ously published observations together, it is possible that the blood type antigens have some roles in oncogenesis and cancer progression in digestive organs. Indeed, an association between blood type A and the incidence of gastric cancer was previously reported $[17,18]$. Interestingly, the presence of the $\mathrm{B}$ antigen was reported to be positively associated with the incidence of ovarian cancer, whereas blood group A was not associated with this risk [19].

When we focus on the pancreas, previous pathological studies demonstrated the deletion and novel expression of the $\mathrm{A}, \mathrm{B}$, and $\mathrm{H}$ antigens on the surface of pancreatic cancer cells compared with the surrounding normal ductal cells [20-23]. It has also been suggested that modifications of the glycosyltransferase specificity occur during pancreatic tumorigenesis [5]. Glycosyltransferases are cell surface molecules that are recognized by the host immune response and may have a role in facilitating immunosurveillance for malignant cells [24,25].

The detailed mechanism connecting the prognosis of pancreatic cancer patients and the ABO blood types remains unknown in the present study. Further explorations of the detailed mechanism may lead to the development of completely new cancer treatments. The mechanism underlying the relationship between blood type A and the effects of gemcitabine adjuvant chemotherapy of pancreatic cancer clearly demonstrated in the present study is also important to overcome the drug tolerance of pancreatic cancer. Further investigations are necessary to elucidate the mechanisms underlying the findings obtained in this study.

\section{Acknowledgements}

We thank Midori Kojyo, Makiko Masuda, and Masayo Matsuzaki (Department of Surgery and Oncology, Kyu- 
shu University) for their secretarial assistance, and the Research Support Center, Graduate School of Medical Sciences, Kyushu University for their technical support.

\section{REFERENCES}

[1] A. Jemal, R. Siegel, E. Ward, Y. Hao, J. Xu and M. J. Thun, “Cancer Statistics," Cancer Journal for Clinicians, Vol. 59, No. 4, 2009, pp. 225-249. http://dx.doi.org/10.3322/caac.20006

[2] A. L. Warshaw and C. Fernandez-del Castillo, "Pancreatic carcinoma," The New England Journal of Medicine, Vol. 326, No. 7, 1992, pp. 455-465. http://dx.doi.org/10.1056/NEJM199202133260706

[3] H. A. Burris, M. J. Moore, J. Andersen, et al., "Improvements in Survival and Clinical Benefit with Gemcitabine as First-Line Therapy for Patients with Advanced Pancreas Cancer: A Randomized Trial,” Journal of Clinical Oncology, Vol. 15, No. 6, 1997, pp. 2403-1413.

[4] D. Li, K. Xie, R.Wolff and J. L. Abbruzzese, "Pancreatic Cancer,” Lancet, Vol. 363, No. 9414, 2004, pp. 10491057. http://dx.doi.org/10.1016/S0140-6736(04)15841-8

[5] B. M. Wolpin, P. Kraft, M. Gross, et al., "Pancreatic Cancer Risk and ABO Blood Group Alleles: Results from the pancreatic Cancer Cohort Consortium," Cancer Research, Vol. 70, No. 3, 2010, pp. 1015-1023. http://dx.doi.org/10.1158/0008-5472.CAN-09-2993

[6] N. Kurata, H. Fujita, K. Ohuchida, et al., "Predicting the Chemosensitivity of Pancreatic Cancer Cells by Quantifying the Expression Levels of Genes Associated with the Metabolism of Gemcitabine and 5-Fluorouracil," International Journal of Oncology, Vol. 39, No. 2, 2011, pp. 473-482.

[7] A. K. Fukunaga, S. Marsh, D. J. Murry, T. D. Hurley and H. L. McLeod, "Identification and Analysis of Single-Nucleotide Polymorphisms in the Gemcitabine Pharmacologic Pathway," The Pharmacogenomics Journal, Vol. 4, No. 5, 2004, pp. 307-314. http://dx.doi.org/10.1038/sj.tpj.6500259

[8] A. W. Blackstock, H. Lightfoot, L. D. Case, et al., "Tumor Uptake and Elimination of 2',2'-Difluoro-2'-Deoxycytidine (Gemcitabine) after Deoxycytidine Kinase Gene Transfer: Correlation with in Vivo Tumor Response," Clinical Cancer Research, Vol. 7, No. 10, 2001, pp. 3263-3268.

[9] M. S. Duxbury, H. Ito, M. J. Zinner, S. W. Ashley and E. E. Whang, "RNA Interference Targeting the M2 Subunit of Ribonucleotide Reductase Enhances Pancreatic Adenocarcinoma Chemosensitivity to Gemcitabine,” Oncogene, Vol. 23, No. 8, 2004, pp. 1539-1548. http://dx.doi.org/10.1038/sj.onc.1207272

[10] H. Fujita, K. Ohuchida, K. Mizumoto, et al., "Gene Expression Levels as Predictive Markers of Outcome in Pancreatic Cancer after Gemcitabine-Based Adjuvant Chemotherapy,” Neoplasia, Vol. 12, No. 10, 2010, pp. 807817.

[11] S. Ohhashi, K. Ohuchida, K. Mizumoto, et al., "DownRegulation of Deoxycytidine Kinase Enhances Acquired
Resistance to Gemcitabine in Pancreatic Cancer," Anticancer Research, Vol. 28, No. 4B, 2008, pp. 2205-2212.

[12] E. Giovannetti, M. Del Tacca, V. Mey, et al., "Transcription Analysis of Human Equilibrative Nucleoside Transporter-1 Predicts Survival in Pancreas Cancer Patients Treated with Gemcitabine,” Cancer Research, Vol. 66, No. 7, 2006, pp. 3928-3935. http://dx.doi.org/10.1158/0008-5472.CAN-05-4203

[13] J. Spratlin, R. Sangha, D. Glubrecht, et al., "The Absence of Human Equilibrative Nucleoside Transporter 1 is Associated with Reduced Survival in Patients with Gemcitabine-Treated Pancreas Adenocarcinoma," Clinical Cancer Research, Vol. 10, No. 20, 2004, pp. 6956-6961. http://dx.doi.org/10.1158/1078-0432.CCR-04-0224

[14] Q. Ben, K. Wang, Y. Yuan and Z. Li, "Pancreatic Cancer Incidence and Outcome in Relation to ABO Blood Groups among Han Chinese Patients: A Case-Control Study," International Journal of Cancer, Vol. 128, No. 5, 2011, pp. 1179-1186. http://dx.doi.org/10.1002/ijc.25426

[15] S. Hakomori, “Antigen Structure and Genetic Basis of Histo-Blood Groups A, B and O: Their Changes Associated with Human Cancer," Biochimica et Biophysica Acta, Vol. 1473, No. 1, 1999, pp. 247-266. http://dx.doi.org/10.1016/S0304-4165(99)00183-X

[16] M. E. Reid and N. Mohandas, "Red Blood Cell Blood Group Antigens: Structure and Function," Seminars in Hematology, Vol. 41, No. 2, 2004, pp. 93-117. http://dx.doi.org/10.1053/j.seminhematol.2004.01.001

[17] I. Aird, H. H. Bentall, J. A. Roberts, "A Relationship between Cancer of Stomach and the ABO Blood Groups,” British Medical Journal, Vol. 1, No. 4814, 1953, pp. 799-801. http://dx.doi.org/10.1136/bmj.1.4814.799

[18] G. Edgren, H. Hjalgrim, K. Rostgaard, et al., "Risk of Gastric Cancer and Peptic Ulcers in Relation to ABO Blood Type: A Cohort Study,” American Journal of Epidemiology, Vol. 172, No. 11, 2010, pp. 1280-1285. http://dx.doi.org/10.1093/aje/kwq299

[19] M. A. Gates, B. M. Wolpin, D. W. Cramer, S. E. Hankinson and S. S. Tworoger, "ABO Blood Group and Incidence of Epithelial Ovarian Cancer,” International Journal of Cancer, Vol. 128, No. 2, 2010, pp. 482-486. http://dx.doi.org/10.1002/ijc.25339

[20] S. H. Itzkowitz, M. Yuan, L. D. Ferrell, et al., "Cancer-Associated Alterations of Blood Group Antigen Expression in the Human Pancreas," Journal of the National Cancer Institute, Vol. 79, No. 3, 1987, pp. 425-434.

[21] E. Uchida, M. A. Tempero, D. A. Burnett, Z. Steplewski, P. M. Pour, "Correlative Studies on Antigenicity of Pancreatic Cancer and Blood Group Types,” Cancer Detection and Prevention, Vol. 1, No., 1987, pp. 145-148.

[22] P. M. Pour, M. M. Tempero, H. Takasaki, et al., "Expression of Blood Group-Related Antigens ABH, Lewis A, Lewis B, Lewis X, Lewis Y, and CA 19-9 in Pancreatic Cancer Cells in Comparison with the Patient's Blood Group Type,” Cancer Research, Vol. 48, No. 19, 1988, pp. 5422-5426.

[23] M. H. Schuessler, S. Pintado, S. Welt, et al., "Blood Group and Blood-Group-Related Antigens in Normal Pancreas and Pancreas Cancer: Enhanced Expression of 
Precursor Type 1, Tn and Sialyl-Tn in Pancreas Cancer,” International Journal of Cancer, Vol. 47, No. 2, 1991, pp. 180-187. http://dx.doi.org/10.1002/ijc.2910470204

[24] S. Zhang, H. S. Zhang, C. Cordon-Cardo, et al., "Selection of Tumor Antigens as Targets for Immune Attack Using Immunohistochemistry: II. Blood Group-Related Antigens,” International Journal of Cancer, Vol. 73, No. 1, 1997, pp. 50-56.
http://dx.doi.org/10.1002/(SICI)1097-0215(19970926)73: 1<50::AID-IJC9>3.3.CO;2-O

[25] S. Hakomori, “Tumor-Associated Carbohydrate Antigens Defining Tumor Malignancy: Basis for Development of Anti-Cancer Vaccines," Advances in Experimental Medicine and Biology, Vol. 491, 2001, pp. 369-402. http://dx.doi.org/10.1007/978-1-4615-1267-7 24 